\title{
A MOBILE COMPUTING TECHNOLOGY FORESIGHT STUDY WITH SCENARIO PLANNING APPROACH
}

\author{
Wei-Hsiu Weng \\ National Chengchi University \\ No.64, Sec. 2, Zhinan Rd., Wenshan District, Taipei City 11605, \\ Taiwan \\ wh.weng@msa.hinet.net \\ Woo-Tsong Lin \\ National Chengchi University \\ No.64, Sec. 2, Zhinan Rd., Wenshan District, Taipei City 11605, \\ Taiwan \\ lin@mis.nccu.edu.tw
}

\begin{abstract}
Although the importance of mobile computing is gradually being recognized, mobile computing technology development and adoption have not been clearly realized. This paper focuses on the technology planning strategy for organizations that have an interest in developing or adopting mobile computing technology. By using scenario analysis, a technology planning strategy is constructed. In this study, thirty mobile computing technologies are classified into six groups, and the importance and risk factors of these technologies are then evaluated under two possible scenarios. The main research findings include the discovery that most mobile computing software technologies are rated high to medium in importance and low risk in both scenarios, and that scenario changes will have less impact on mobile computing devices and on mobile computing software technologies. These results provide a reference for organizations interested in developing or adopting mobile computing technology.
\end{abstract}

Keywords: Mobile Computing, Scenario Analysis, Technology Foresight, Strategy

\section{INTRODUCTION}

Mobile computing has grown in popularity with the development of innovative smart mobile devices such as smart phones and tablets. 
Businesses have adopted mobile computing technology and migrated their computing resources from their own server rooms to mobile service providers. Mobile device users are now creating and sharing content instead of just browsing, and thanks to the popularity of mobile social services, it is easier than ever to share and access information. Users have therefore been encouraged to move their data from personal computers to the data centers of mobile service providers. To offer more reliable and responsive service, mobile service providers must upgrade their computing capacity and storage space and modify all new mobile service architectures to meet user requirements.

Firms worldwide are now exploring possible opportunities into the mobile computing market. However, what is the scope of mobile computing technology? What are the outlooks in terms of the importance and risks of these technologies? These key questions need to be answered before firms can have confidence in the accuracy of mobile business strategy planning.

\section{LITERATURE REVIEW}

Mobile computing technology has evolved from the popularization of smartphones such as the iPhone. When Apple released its first-generation iPhone with the iOS system in 2007, Google released the open embedded operating system Android for smart mobile devices, and has cooperated with other mobile phone manufacturers to establish the Open Handset Alliance $^{1}$, which brought drastic changes to the smartphone market ${ }^{2}$ that had centered on Nokia and RIM, resulting in a paradigm shift, and the development of the mobile application software market. With the rise of cloud computing and the growth of the mobile application software market, it provided a stage for IT industry to expand its mobile computing technologies ${ }^{3}$.

Since the added value created by hardware is more limited, important global companies have started to invest in mobile application software; and in order to satisfy user experience they have increased the added value for products, mobile application software (Mobile App) has become the key competition for each company to differentiate its products ${ }^{4,5}$.

In order to establish ubiquitous mobile computing, the infrastructure of communication networks need to be constructed. Currently, several networking infrastructure are under development, such as IoT (Internet of Things) ${ }^{6,7}$ and wireless sensor network ${ }^{8}$. Kim and Feamster ${ }^{9}$ discussed the concept and advancement of software defined network (SDN), which is a technology for management flexibility of data center networking devices. 
Mobile user devices are terminal devices for end users of mobile computing applications. Leading IT companies such as Amazon, Apple, Google and Microsoft have developed innovative mobile devices such as the smartphone and tablet, and have expanded the mobile computing software market of smart mobile devices ${ }^{10}$. Smart wearable devices are the next mobile computing devices with great market potential ${ }^{11}$.

Remote sensing technologies provide interconnecting functions between mobile devices. Near Field Communication (NFC) ${ }^{12}, 13$ evolved from Radio Frequency Identification (RFID) $)^{14,15,16}$ and interconnection technology. In the past, non-contact chips were produced as card applications. However, chips are now embedded into mobile devices for greater convenience. Mobile devices have become payment tools that permit the downloading and payment of services in any public setting and the exchange of data on mobile devices.

The convenience provided by mobile applications also caused security problems with mobile data. Mobile data security technology has two dimensions. One dimension is the adoption of IT security technology, products or services by businesses to improve the security of mobile services. This is "Security for Cloud" "17, 18 . The other dimension is the use of security technology by IT security vendors to strengthen, expand or transform their existing IT security products and services. This is "Security as a Service." BYOD (Bring Your Own Device) and MDM (Mobile Device Management) are now the main issues for many enterprises adopting mobile devices $^{19,20}$.

Finally, mobile application software on mobile phones and tablets become more popular, more mobile development and deployment tool will be linked to the app market ${ }^{21}$. This trend will see more software developers release innovative applications that link apps to SoLoMo (Social, Location-based, Mobile) services, thus creating new opportunities for software vendors.

\section{RESEARCH METHOD}

Scenario Analysis ${ }^{22}$ has been applied in various domains to forecast trends in technology. Different versions scenario analysis methods have been proposed ${ }^{23}$. It is used in technology portfolio planning process ${ }^{24}$ to assist in the strategic decision for finding the feasible resource allocation plans that best fit organizational goals. Scenario Analysis is used by futurists to develop future situations in order to facilitate strategic action plans and to create visions for the future.

The key steps of scenario analysis are as follows. 
1. Identify significant impact variables for scenario construction.

2. Propose possible scenarios by exploring combinations of impact variables.

3. Construct a set of technology alternatives and classify them into groups.

4. Generate a set of technology assessment indicators.

5. Evaluate the technology alternatives by technology assessment indicators.

To conduct the technology foresight study, an expert panel was selected with eleven domain experts selected from the IT industry. This expert panel consisted of the following members:

1. Three consultant managers of publicly listed IT services firms.

2. Four CEO and VP level executives of independent software vendors.

3. Two R\&D managers of publicly listed IT device manufacturers.

4. Two project managers of publicly listed telecom operators.

A facilitator led the expert panel discussion sessions. Activities in these sessions included open discussions, anonymous voting, as well as the administration of surveys.

\section{RESULTS}

To identify decision-making criteria, expert discussions were conducted concerning decision factors from the social, technological, economic and political perspectives. Possible factors were elaborated, such as the global market outlook, technology breakthrough, and competition within the industry. The final set of indicators is summarized in Table 1.

Impact variables most likely to affect the scenario development were identified by the expert panel. Through evaluations from different combinations of these variables, final choices of scenarios were proposed. The scenarios were labeled and elaborated upon. The results are illustrated in Table 1.

Mobile computing technologies were collected by interviewing experts and from secondary data which included vendor propositions and research literature. The most promising mobile computing technologies are summarized in Table 2. 
Table 1. Proposed scenarios under impact variables

\begin{tabular}{ccccc}
\hline $\begin{array}{c}\text { Global Market } \\
\text { Outlook }\end{array}$ & $\begin{array}{c}\text { Technology } \\
\text { Breakthrough }\end{array}$ & $\begin{array}{c}\text { Vendor } \\
\text { Competition }\end{array}$ & $\begin{array}{c}\text { Scenario } \\
\text { Encoding }\end{array}$ & $\begin{array}{c}\text { Selected Scenario } \\
\text { and Naming }\end{array}$ \\
\hline Good & Fast & High & 000 & Big Demand \\
Poor & Fast & High & 100 & \\
Good & Slow & High & 010 & Pessimistic \\
Poor & Slow & High & 110 & \\
Good & Fast & Low & 001 & \\
Poor & Fast & Low & 101 & \\
Good & Slow & Low & 011 & \\
Poor & Slow & Low & 111 & \\
\hline
\end{tabular}

Table 2. Mobile technology alternatives

\begin{tabular}{cc}
\hline Classification & Technology \\
\hline & MCS1: Mobile app \\
Mobile Computing Software & MCS2: Location based service \\
(MCS) & MCS3: Mobile social media \\
& MCS4: Mobile big data analytics \\
MCS5: Mobile OS and app store \\
\hline & MCN1: Internet of Thing (IoT) \\
MCN2: Smart grid \\
Mobile Communication & MCN3: Wireless sensor network \\
Network & MCN4: 4G/5G infrastructure \\
(MCN) & MCD1: Smartphone \\
& MCD2: Tablet \\
& MCD3: Wearable device \\
Mobile Computing Device & MCD4: Smart automobile \\
(MCD) & MCD5: Robot \\
\hline & MCA1: RFID application \\
Mobile Cognizant Application & MCA2: QR code application \\
(MCA) & MCA3: NFC application \\
& MCA4: Voice recognition \\
& MCA5: Gesture recognition \\
\hline & MDS1: BYOD solution \\
Mobile Data Security & MDS2: MDM solution \\
(MDS) & MDS3: Security as a service \\
& MDS4: Security for cloud \\
& MDS5: Bio-Identification \\
\hline & MDD1: Platform as a service (PaaS) \\
Mobile Development and & MDD2: Middleware for mobile solution \\
Deployment Tool & MDD3: Enterprise app store \\
(MDD) & MDD4: Rich Internet application (RIA) \\
& MDD5: HTML5 \\
\hline
\end{tabular}


The expert panel then determined the Importance and Risk indicators for the assessment of technology. These indicators are summarized in Table 3.

Table 3. Technology assessment indicators

\begin{tabular}{clccc}
\hline Dimension & \multicolumn{1}{c}{ Indicator } & $\begin{array}{c}\text { Low } \\
\text { Value }\end{array}$ & $\begin{array}{c}\text { Medium } \\
\text { Value }\end{array}$ & $\begin{array}{c}\text { High } \\
\text { Value }\end{array}$ \\
\hline \multirow{2}{*}{ Importance } & $\begin{array}{l}\text { Annual growth rate of } \\
\text { global market size for } \\
\text { the next 5 years }\end{array}$ & $<10 \%$ & $10 \% \sim 20 \%$ & $>20 \%$ \\
\cline { 2 - 5 } & $\begin{array}{l}\text { Global user adoption } \\
\text { ratio }\end{array}$ & $<10 \%$ & $10 \% \sim 60 \%$ & $>60 \%$ \\
\hline \multirow{2}{*}{ Risk } & $\begin{array}{l}\text { Annual growth rate of } \\
\text { local production value } \\
\text { for the next 5 years }\end{array}$ & $>10 \%$ & $10 \% \sim 5 \%$ & $<5 \%$ \\
\cline { 2 - 5 } & $\begin{array}{l}\text { Local R\&D over revenue } \\
\text { proportion }\end{array}$ & $>9 \%$ & $9 \% \sim 3 \%$ & $<3 \%$ \\
\hline
\end{tabular}

Based on the important indicators and risk indicators in Table 3, the expert panel assessed the mobile computing technologies compiled in Table 2 with respect to the two scenarios. The assessment results are exhibited in Figure 1 and Figure 2.

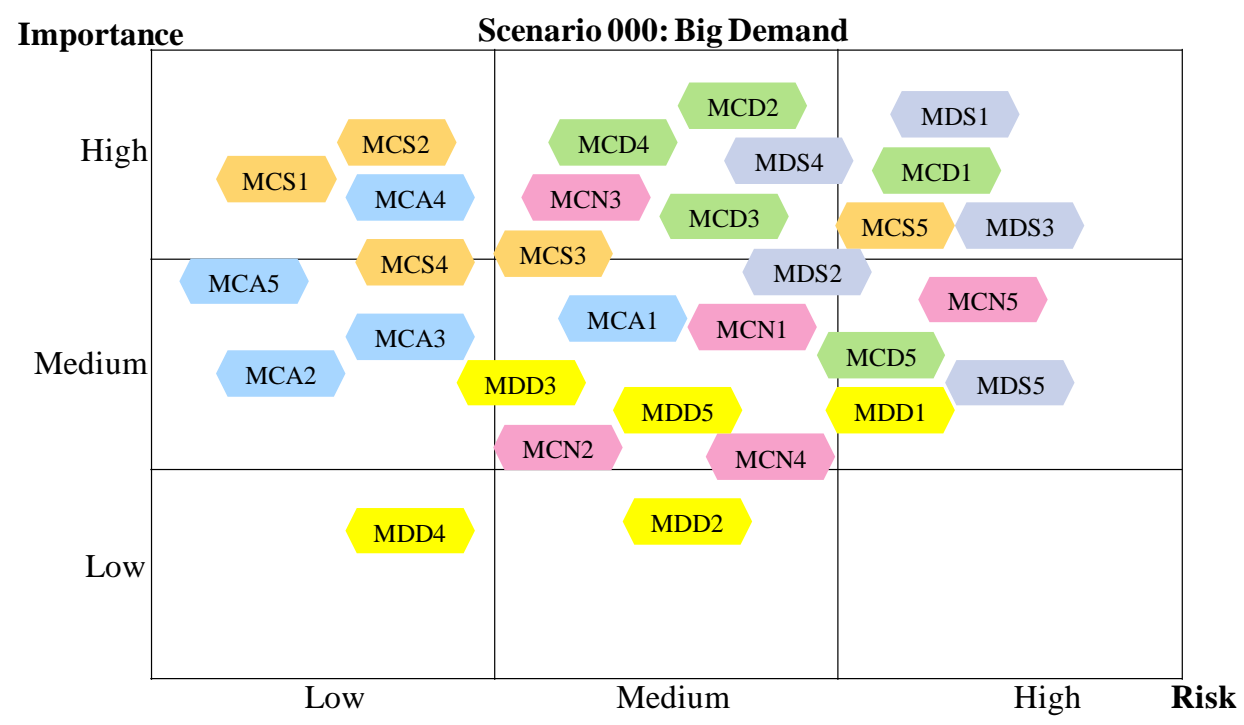

Figure 1. Technology assessment for Big Demand Scenario 


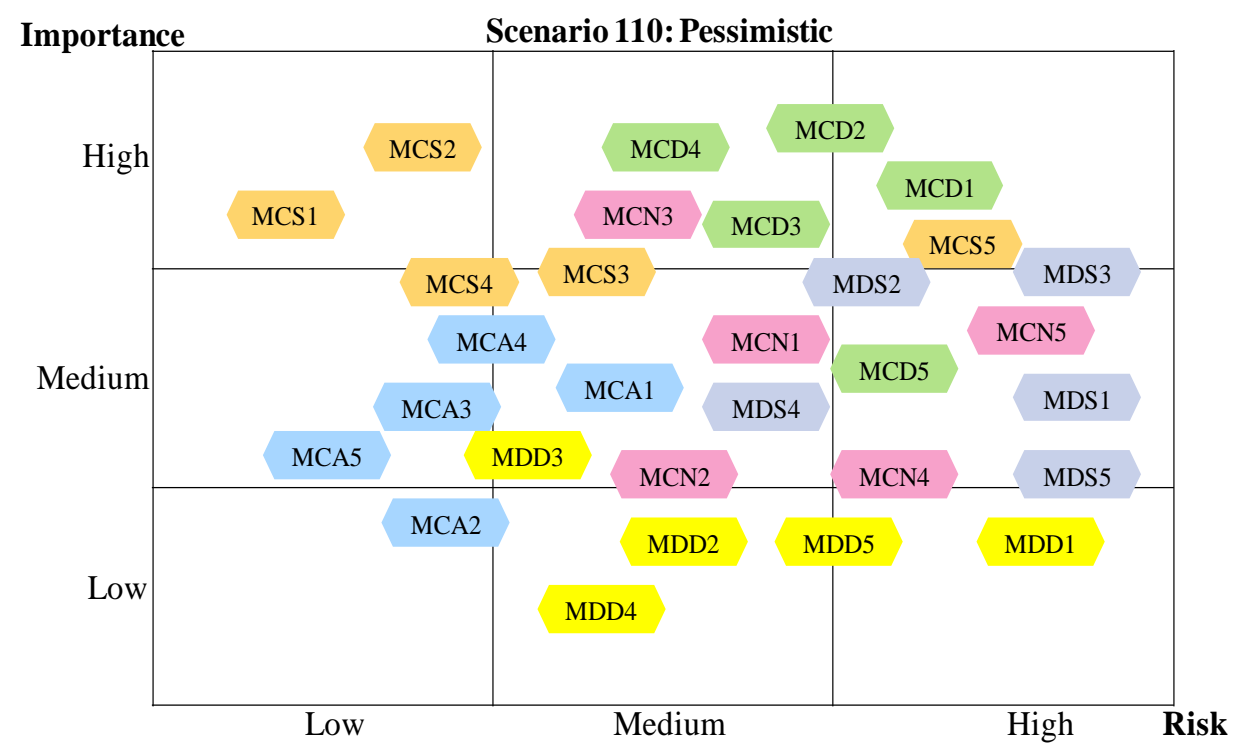

Figure 2. Technology assessment for Pessimistic Scenario

\section{CONCLUSIONS}

Based on these findings, strategic thinking of an organization for developing or adopting mobile computing technology for advantages can be initiated in the pursuit of new market opportunities with low risks.

For the Big Demand scenario, the mobile computing software (MCS) technologies are generally of high importance and low or medium risk. This observation is consistent with the fact that the mobile computing software technologies, based on SoLoMo (social, location based and mobile) applications, are gaining industry support and a large base of users worldwide.

For the Pessimistic scenario, the global market outlook is poor. The advancement of mobile computing technology is slowing. The risk rating of most mobile computing related technologies would rise and their importance rating would drop. An important observation is that the mobile computing software (MCS) and mobile computing device (MCD) technologies are most robust against scenario changes. This suggests that these technologies are suitable for vulnerable enterprises to adopt.

\section{REFERENCES}

[1] J. Smajda, Open source and the moral field of computing. Journal of Information Technology \& Politics, 8(3), p304-322, 2011. http://dx.doi.org/10.1080/19331681.2011.595333. 
[2] G. Gueguen and T. Isckia, The borders of mobile handset ecosystems: Is coopetition inevitable? Telematics and Informatics, 28(1), p5-11, 2011. http://dx.doi.org/10.1016/j.tele.2010.05.007.

[3] H. Bouwman, S. Dihal, M. de Reuver, M. Warnier and C. Carlsson, Mobile cloud computing: State of the art and outlook. info, 15(1), p4-16, 2013. http://dx.doi.org/10.1108/14636691311296174.

[4] C. Watson, J. McCarthy, and J. Rowley, Consumer attitudes towards mobile marketing in the smart phone era. International Journal of Information Management, 33(5), p840-849, 2013. http://dx.doi.org/10.1016/j.ijinfomgt.2013.06.004.

[5] L.A. Mills, G. Knezek, and F. Khaddage, Information seeking, information sharing, and going mobile: Three bridges to informal learning. Computers in Human Behavior, 32, p324-334, 2014. http://dx.doi.org/10.1016/j.chb.2013.08.008.

[6] L. Atzori, A. Iera, and G. Morabito, The Internet of Things: A survey. Computer Networks, 54(15), p2787-2805, 2010. http://dx.doi.org/10.1016/j.comnet.2010.05.010.

[7] C. Lerche, K. Hartke, and M. Kovatsch, Industry adoption of the Internet of Things: A constrained application protocol survey. Paper presented at the International Conference on Emerging Technologies \& Factory Automation, Krakow, Poland, September 17-21, 2012. http://dx.doi.org/10.1109/ETFA.2012.6489787.

[8] M.J. Piran, G.R. Murthy, and G.P. Babu, Vehicular ad hoc and sensor networks principles and challenges. International Journal of Ad hoc, Sensor \& Ubiquitous Computing, 2(2), p38-49, 2011.

[9] H. Kim, and N. Feamster, Improving network management with Software Defined Networking. IEEE Communications Magazine, 51(2), p114 -119, 2013. http://dx.doi.org/10.1109/MCOM.2013.6461195.

[10] A. Holzer, and J. Ondrus, Mobile application market: A developer's perspective. Telematics and Informatics, 28(1), p22-31, 2011. http://dx.doi.org/10.1016/j.tele.2010.05.006.

[11] M. Chana, D. Estèvea, J.Y. Fourniolsa, C. Escribaa, and E. Campoa, Smart wearable systems: Current status and future challenges. Artificial Intelligence in Medicine, 56(3), p137-156, 2012. http://dx.doi.org/10.1016/j.artmed.2012.09.003.

[12] G.W.H. Tan, K.B. Ooi, S.C. Chong, and T.S. Hew, NFC mobile credit card: The next frontier of mobile payment? Telematics and Informatics, 31(2), p292-307, 2014. http://dx.doi.org/10.1016/j.tele.2013.06.002.

[13] L.Y. Leong, T.S. Hew, G.W.H. Tan, and K.B. Ooi, Predicting the determinants of the NFC-enabled mobile credit card acceptance: A neural networks approach. Expert Systems with Applications, 40(14), p5604-5620, 2013. http://dx.doi.org/10.1016/j.eswa.2013.04.018. 
[14] E. Ilie-Zudor, Z. Kemény, F. van Blommestein, L. Monostori, and A. van der Meulen, A survey of applications and requirements of unique identification systems and RFID techniques. Computers in Industry, 62(3), p227-252,

2011. http://dx.doi.org/10.1016/j.compind.2010.10.004.

[15] E.W.T. Ngai, K.K.L. Moon, F.J. Riggins, and C.Y. Yi, RFID research: An academic literature review (1995-2005) and future research directions. International Journal Production Economics, 112(2), p510-520, 2008. http://dx.doi.org/10.1016/j.ijpe.2007.05.004.

[16] F.J. Villanueva, D. Villa, F. Moya, M.J. Santofimia, and J.C. Lopez, Internet of things architecture for an RFID-based product tracking business model. Paper Presented at the International Conference on Innovative Mobile and Internet Services in Ubiquitous Computing, Palermo, Italy, July 4-6, 2012. http://dx.doi.org/10.1109/IMIS.2012.59.

[17] S. Sengupta, An approach to provide a network layer security model with QR code generated with shuffled GPS parameters as embedded keys traveling over Internet using existing IPv4 mechanism. Computer Networks, 57(11), p2313-2330, 2013. http://dx.doi.org/10.1016/j.comnet.2013.04.003.

[18] S. Subashini, and V. Kavitha, A survey on security issues in service delivery models of cloud computing. Journal of Network and Computer Applications, 34(1), p1-11, 2011. http://dx.doi.org/10.1016/j.jnca.2010.07.006.

[19] G.P. Hancke, K. Markantonakis, and K.E. Mayes, Security challenges for user-oriented RFID applications within the "Internet of things". Journal of Internet Technology, 11(3), p307-313, 2010.

[20] S. Shin, K. Kim, K.-H. Kim, and H. Yeh, A remote user authentication scheme with anonymity for mobile devices. International Journal of Advanced Robotic Systems, 9, p1-8, 2012. http://dx.doi.org/10.5772/50912.

[21] N. Serrano, J. Hernantes, and G. Gallardo, Mobile Web Apps. IEEE Software, 30(5), p22-27, 2013. http://dx.doi.org/10.1109/MS.2013.111.

[22] SRI, Integrated Technology Planning. SRI International Report, Menlo Park, California, 1996.

[23] D. Mietzner, and G. Reger, Advantages and disadvantages of scenario approaches for strategic foresight. International Journal of Technology Intelligence and Planning, 1(2), p220-239, 2005.

[24] O.S. Yu, Technology portfolio planning and management: Practical concepts and tools. New York: Springer, 2006. 
